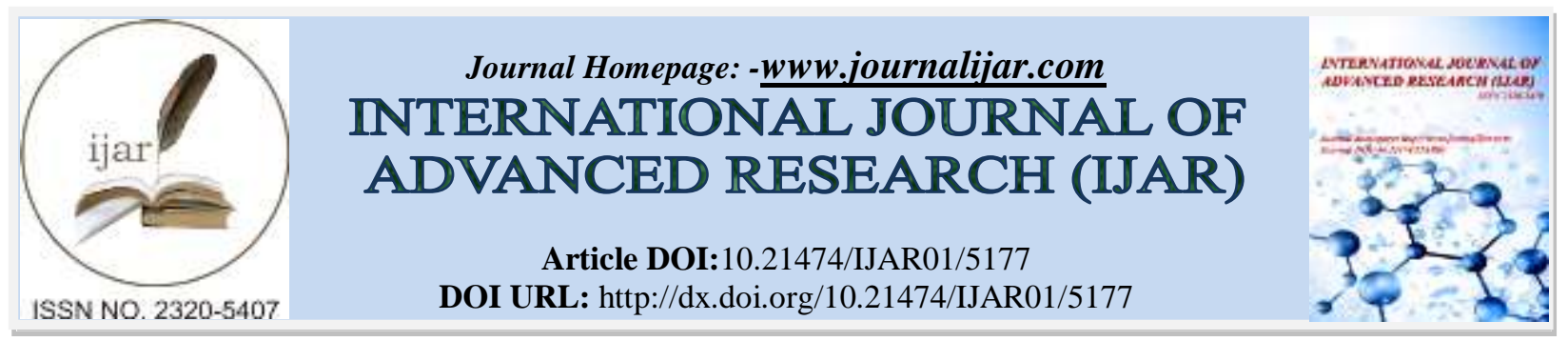

RESEARCH ARTICLE

\title{
ASSOCIATION BETWEEN FUNGIFORM PAPILLAE DENSITY AND BODY MASS INDEX.
}

\section{Dr. Subash B.V ${ }^{1}$, Dr. Asha R Iyengar ${ }^{2}$, Dr. Vani M.H ${ }^{3}$, Dr. Seema Patil ${ }^{4}$ and Dr. Revan Kumar Joshi ${ }^{5}$.}

1. Reader, Department of Oral medicine and Radiology, D.A.P.M.R.V Dental College, Bangalore, India.

2. Professor and HOD, Department of Oral Medicine and Radiology, D.A.P.M.R.V Dental College, Bangalore, India.

3. Post graduate student, Department of Oral Medicine and Radiology, D.A.P.M.R.V Dental College, Bangalore, India.

4. Reader, Department of Oral medicine and Radiology, D.A.P.M.R.V Dental College, Bangalore, India.

5. Lecturer, Department of Oral Medicine and Radiology, D.A.P.M.R.V Dental College, Bangalore, India.

\section{Manuscript Info}

(.........................

Manuscript History

Received: 15 June 2017

Final Accepted: 17 July 2017

Published: August 2017

Key words:-

Fungiform papillae density, BMI, sweets, fatty foods, obesity

\section{Abstract}

Background and objectives: Obese subjects have an inherent chemosensory deficit due to reduced Fungiform papillae density (FPD) resulting in increased food consumption. The study aimed to assess the relationship between Body mass index (BMI) and Fungiform papillae density in subjects with increased and normal BMI.

Methodology: A total of 110 subjects were randomly included in the study. The BMI of the subjects was calculated after recording the height and weight of the subjects. Subjects were categorized as Normal weight (18.0 to $22.9 \mathrm{~kg} / \mathrm{m}^{2}$ ), Overweight (23.0 to $24.9 \mathrm{~kg} / \mathrm{m}^{2}$ ) and Obese (Greater than $25 \mathrm{~kg} / \mathrm{m}^{2}$ ) based on the standard BMI definition values of Asian Indian population. The FPD was assessed on a photograph of a stained area of the tongue. Scoring of fungiform papillae was done using the Denver Papillae Protocol Dichotomous Key and counted using a software.Results were tabulated and statistically analyzed.

Results: There was a significant negative correlation between BMI and FPD (correlation $=-0.344, \mathrm{p}=0.000$ ) among the normal weight, overweight and obese subjects. Multiple regression analysis revealed a significant reduction in FPD with increasing frequency of eating sweets and fatty foods ( $\mathrm{p}=0.028$ and 0.023 respectively).

Conclusion: A reduction in FPD was noted with increase in BMI which in turn may have a role in food consumption pattern of an individual.

Copy Right, IJAR, 2017,. All rights reserved.

\section{Introduction:-}

Taste is a chemical sense that is activated during eating, drinking, and tasting of foods in humans (Frank etal.,1992).Taste buds are the peripheral sensory organs that respond to the taste stimuli and form an integral component of the taste system. The quantification of taste buds is very important in the evaluation of sense of taste (Webb etal.,2015). 
Fungiform Papillae (FP) in humans contain at least one taste bud and the Fungiform papillae density (FPD) is positively related to taste sensitivity (Segovia etal.,2002). The FPD in a small area over the anterior tongue dorsum is proven to be representative of the overall papillae number or density (Correa etal.,2013). The taste buds in the FP contain fatty acid receptors and mechanoreceptors. Thus, a higher amount of FP may not only increase the fat perception, but also increases the taste acuity in general (Proserpio etal.,2015).

Obesity has been associated with diet containing high levels of both fat and sucrose. Obese subjects differ in taste sensitivity from normal weight subjects and these differences might lead to different food preferences. Obese subjects seem to prefer energy dense food more than normal weight subjects (Proserpio etal.,2015).More specifically, obese subjects seem to be less sensitive to taste stimuli, especially with fatty acid chemoreception, and have a reduced number of FP and hence may have an increased need for food to compensate for their chemosensory deficit (Donaldson etal.,2009).

Racial and ethnic variations have an important role in obesity and also in FPD. Most of the studies exploring the relationship between FPD and obesity were done in European and American population (Proserpio etal.,2015 and Donaldson etal.,2009). The conclusions drawn from these studies cannot be generalized for the Indian population.

Hence the present study was aimed to assess the relationship between Fungiform papillae density and body mass index in subjects with increased and normal BMI, in a subset of Indian population.

\section{Methodology:-}

The study was a prospective comparative study. The subjects for the study were selected from the outpatient department of Oral Medicine and Radiology. A total of 110 subjects were included in the study out of which 50 subjects had increased BMI and 60 subjects had normal BMI.

Subjects of age group between 20 to 49 years were included in the study. Subjects having systemic conditions(Pavlidis etal.,2014 and Scott etal.,1985) and on medications(Fischer etal.,2013) affecting FPD, pregnant and lactating women, subjects with any obvious mental illness and those with habits of smoking, tobacco and betel quid chewing and alcohol consumption (Fischer etal.,2013)were excluded from the study.

The study was approved by the institutional ethics committee.Prior to conducting the study, an informed consent was obtained from all the subjects selected for the study. A detailed case history was recorded with emphasis on the factors influencing BMI such as dietary habits, life style and physical activity. Body mass index was calculated for all the subjects. The tongue was examined for evaluating the Fungiform papillae density.

\section{Calculation of the Body Mass index:-}

The participant's height was measured using a height chart and measurements were recorded to the nearest meter. Weight of the participants was measured using manual weighing machine and recorded to the nearest kilogram.

BMI was calculated using the equation: Weight $(\mathrm{kg}) / \mathrm{Height}^{2}\left(\mathrm{~m}^{2}\right)$. The subjects were categorized based on the standard BMI definition values of Asian Indian population(Misra etal.,2009) as Normal weight (18.0 to $22.9 \mathrm{~kg} / \mathrm{m}^{2}$ ), Overweight (23.0 to $24.9 \mathrm{~kg} / \mathrm{m}^{2}$ ) and Obese (Greater than $25.0 \mathrm{~kg} / \mathrm{m}^{2}$ ).

\section{Fungiform papillae density evaluation:-}

After rinsing the mouth with water and drying the tongue, a cotton bud dipped into toluidine blue stain was used to transfer a small amount of the stain onto the anterior dorsal surface of tongue, immediately left of the midline of the tongue and close to the tip. Once the excess dye was removed by rinsing, the subjects were directed to position themselves with their tongue extended a comfortable distance and secured gently between their teeth.

A $2 \mathrm{~cm} \mathrm{X} 2 \mathrm{~cm}$ piece of square filter paper with an $8 \mathrm{~mm}$ diameter circular cutout punched on it and the participant's identification number labeled was placed on the stained part of the tongue next to midline. Three close-up photographs of the tongue, capturing the entire $8 \mathrm{~mm}$ circular stained area on the tongue was obtained using Canon Power Shot A650 IS digital camera. The photos were uploaded to a computer and analyzed using image J software (Fig 1).

A standard magnification of $33.3 \%$ to $50 \%$ was used to ensure consistency between scorers and for use in subsequent steps to score FP. Scoring of FP was done using the Denver Papillae Protocol Dichotomous Key wherein 
Fungiform papillae were identified based on the shape, color, size and recession characteristics and counted on the cell counter(Nuessle etal.,2015).

Each image was scored by two examiners independently, after obtaining a consensus between them, to avoid bias. The images were also evaluated by one of the observers for the second time after 1 week. All results were tabulated and statistically analyzed.

For statistical analysis, the subjects were also categorized as those consuming sweets or fatty foods less than 7 times in a week, 7 times in a week and more than 7 times in a week.

\section{Statistical tests used:-}

Pearson's correlation coefficient was used to determine the association between FPD and BMI in the study subjects. Multiple Regression analysis was used to determine the association of FPD with increasing age, gender and the frequency of eating sweets and fatty foods. Kappa test was used to assess intra observer and inter observer variability.

\section{Results:-}

A total of 110 subjects were included in the study with an age range of 20 to 49 years (Mean age was 31.3years) and of these, $44(40 \%)$ were males and $66(60 \%)$ were females. There was no significant difference in mean age between males and females in the present study (Mean age was 30.9years in males and 31.6years in females).

The inter observer agreement was $66.7 \%$ which was considered as a moderate agreement between the two observers and the intra observer agreement was $85.5 \%$ which was considered as good agreement between the scorings.

FPD was estimated in all the study subjects as the number of papillae per $\mathrm{cm}^{2}$ area $\left(\mathrm{pap} / \mathrm{cm}^{2}\right)$. In the study subjects the FPD ranged from 2 to $50 \mathrm{pap} / \mathrm{cm}^{2}$.

The mean FPD was estimated in different age groups and among males and females. There was no significant difference in the mean FPD between the gender and in different age groups ( $\mathrm{p}=0.682$ and $\mathrm{p}=0.668$ respectively).

The study subjects were categorized into those who where normal weight, those who were overweight and those who were obese based on their calculated BMI. Out of the 110 subjects, 60(54.54\%) were of normal weight, $16(14.54 \%)$ were overweight and 34(30.91\%) were obese.

Table 1 summarizes the comparison of FPD with BMI. On statistical analysis, there was a significant small negative correlation between BMI and FPD (correlation= $-0.344, \mathrm{p}=0.000$ ) among the normal weight, overweight and obese subjects. Thus a reduction in the FPD with increase in BMI was noted.

Table 2 summarizes the comparison of the frequency of sweet and fatty food consumption with FPD. Multiple regression analysis revealed a significant reduction in FPD with increasing frequency of eating sweets and fatty foods ( $\mathrm{p}=0.028$ and 0.023 respectively), with the fatty foods having a greater influence (beta value $=0.216$ ).

\section{Discussion:-}

Obesity, which was a global epidemic, has now become a pandemic. Asian Indians exhibit unique features of obesity; excess body fat, abdominal adiposity, increased subcutaneous and intra-abdominal fat and deposition of fat in ectopic sites such as liver and muscle (Misra etal.,2009).Based on percentage body fat and morbidity data, limits of normal BMI are narrower and lower in Asian Indians as compared to other populations (Misra etal.,2009).In the present study, the 110 study subjects were grouped into normal weight, overweight and obese categories based on the Asian Indian cutoff values(Misra etal.,2009).

Obesity has a multifactorial etiology with dietary habits of an individual playing an important role. Eating behavior of a person depends on gustatory receptors located on the tongue papillae. Among the tongue papillae, FP are regarded as relatively stable anatomical structures and are considered as the original genetic component in taste(Bartoshuk etal.,2000). 
Studies have demonstrated a possible relation between FPD and BMI, with a reduction in FPD with increase in BMI (Proserpio etal.,2015; Hayes etal.,2007 and Essick etal.,2003).The results of the present study was in accordance with that of the studies involving American population(Hayes etal.,2007) and among the Asian and Caucasian populations (Essick etal.,2003).Studies have shown that FP is positively associated with taste sensitivity, especially of the sweet and fat taste perception (Proserpio etal.,2015). Hence reduced FP may lead to reduced diet satisfaction and a tendency for increased food consumption and ultimately weight gain (Proserpio etal.,2015).

To further understand the relation between FPD and BMI, the present study, included the comparison of sweets and fatty foods consumption pattern with FPD. The mean FPD was found to be reduced with the group having increased frequency of eating sweets and fatty foods. These results in the present study were in accordance with studies conducted in United States(Salbe etal.,2004) and in Italian population (Proserpio etal.,2015).

The threshold levels for the perception of sweet, bitter, sour, salty and fats are found to be much higher in obese subjects as compared to non obese(Proserpio etal.,2015).Obese subjects show an increased likeness for sweet and fatty foods than non obese and prefer fat more than sweet. Decreased perception of sweetness in obese could contribute to increased preference for fat because decreased intensity of one orosensory system (ie sweet perception) can compensatorily activate another orosensory system (ie fat preferences)(Bartoshuk etal.,2006).For both normal weight and for obese subjects, the palatability or pleasantness of the food is more when fat and sugar are combined. Such preferences stimulate consumption even without a feeling of hunger. This can lead to excessive food consumption and weight gain(Montmayeur etal.,2010). It has also been proposed that obese subjects are more reluctant to taste new varieties of food and hence are more prone towards energy dense and nutritionally poor dietary habits contributing to increased BMI (Laureati etal.,2015).

\section{Conclusion:-}

It could be concluded that a reduction in FPD is noted with increasing BMI and an increase in the frequency of sweet and fatty food consumption. Reduced FPD may lead to inadequate diet satisfaction and altered taste perception, especially to sweet and fatty foods, which in turn may contribute to an increased consumption of high energy foods resulting in weight gain.

FPD evaluation gives an insight not only to the taste perception of an individual but also points out to the inherent chemosensory deficit in an individual which may contribute to obesity. Hence this should be considered while providing holistic treatment for obesity which includes nutritional or diet counseling apart from pharmacologic therapy.

Table 1:- Mean FPD in different categories of BMI

\begin{tabular}{|l|l|l|}
\hline & $\begin{array}{l}\text { Range } \\
\left(\mathrm{pap} / \mathrm{cm}^{2}\right)\end{array}$ & $\begin{array}{l}\text { Mean } \pm \mathrm{SD} \\
\left(\mathrm{pap} / \mathrm{cm}^{2}\right)\end{array}$ \\
\hline Normal weight & $2-50$ & $19.52 \pm 10.2$ \\
\hline Over weight & $6-34$ & $17.5 \pm 10.1$ \\
\hline Obese & $2-26$ & $11.12 \pm 7.62$ \\
\hline
\end{tabular}

Correlation $=-0.344, \mathrm{p}<0.05^{*}(\mathrm{p}=0.000)$

The table shows a significant negative correlation between Fungiform papillae density (FPD) and body mass index (BMI) (correlation=-0.344). FPD is indicated as the number of papillae per $\mathrm{cm}^{2}$ area $\left(\mathrm{pap} / \mathrm{cm}^{2}\right)$.

Table 2:- The mean FPD vs frequency of eating sweets and fatty foods

\begin{tabular}{|l|l|l|}
\hline Frequency of consumption & Sweets & Fatty foods \\
\hline$<7$ times/wk & $17.38 \pm 10.08$ & $17.4 \pm 10.02$ \\
\hline 7 times/wk & $16.18 \pm 10.02$ & $17.58 \pm 10.12$ \\
\hline$>7$ times/wk & 11 & $10 \pm 5.9$ \\
\hline p value & $0.028^{*}$ & $0.023^{*}$ \\
\hline
\end{tabular}

$\mathrm{P}<0.05^{*}$

The table shows reduction in Fungiform papillae density (FPD) with increase in the frequency of eating sweets and fatty foods. 
Figure 1:-Scoring of FPD using Image $\mathbf{J}$ software

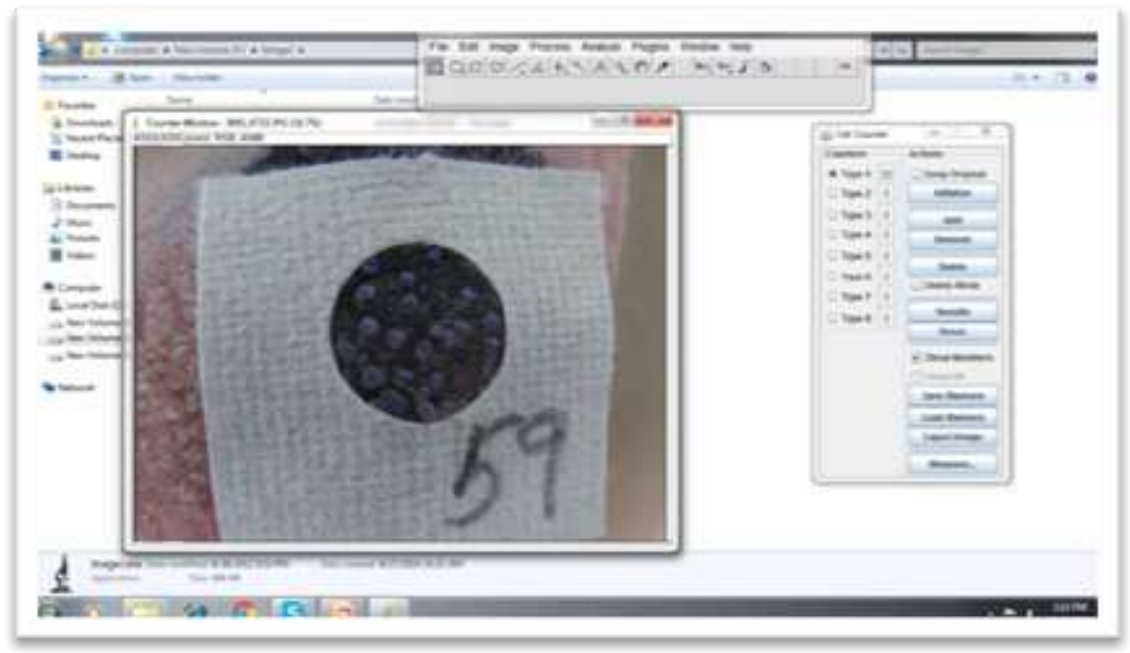

\section{References:-}

1. Bartoshuk LM (2000): Comparing sensory experiences across individuals: Recent psychophysical advances illuminate genetic variation in taste perception. Chem Senses; 25:447-460.

2. Bartoshuk LM, Duffy VB, Hayes JE, Moskowitz HR, Snyder DJ (2006): Psychophysics of sweet and fat perception in obesity: problems, solutions and new perspectives. Phil Trans R Soc B; 361:1137-1148.

3. Correa M, Hutchinson I, Laing DG, Jinks AL (2013): Changes in Fungiform Papillae Density During Development in Humans. Chem Senses; 38: 519-527.

4. Donaldson LF, Bennett L, Baic S, Melichar JK (2009): Taste and weight: Is there a link? Am J ClinNutr; 90(suppl): 800S-3S.

5. Essick GK, Chopra A, Guest S, McGlone F (2003):Lingual tactile acuity, taste perception, and the density and diameter of fungiform papillae in female subjects. Physiol Behav;80: 289- 302.

6. Fischer M E,Cruickshanks KJ, Schubert CR, Pinto A, Klein R, Pankratz N et al (2013): Factors related to fungiform papillae density: The Beaver Dam Offspring Study. Chem Senses; 38(8): 669-677.

7. Frank ME, Hettinger TP, Mott AE (1992): The Sense of Taste: Neurobiology, Aging, and Medication Effects. Crit Rev Oral Biol Med; 3(4):371-393.

8. Hayes JE, Duffy VB (2007): Revisiting sugar-fat mixtures: sweetness and creaminess vary with phenotypic markers of oral sensation. Chem Senses;32(3):225-236.

9. Laureati M, Bertoli S, Bergamaschi V, Leone A, Lewandowski L, Giussani B etal (2015): Food neophobia and liking for fruits and vegetables are not related to excess weight in Italian children. Food Qual Prefer; 40:125-131.

10. Misra A, Chowbey P, Makkar BM, Vikram NK, Wasir JS, Chadha D etal (2009): Consensus Statement for Diagnosis of Obesity, Abdominal Obesity and the Metabolic Syndrome for Asian Indians and Recommendations for Physical Activity, Medical and Surgical Management. J Assoc Physicians India; 57: 163-170.

11. Montmayeur JP, le Coutre J (2010): Fat Detection: Taste, Texture, and Post Ingestive Effects. Boca Raton (FL): CRC Press/Taylor \& Francis.

12. Nuessle TM, Garneau NL, Sloan MM, Santorico SA (2015): Denver Papillae Protocol for Objective Analysis of Fungiform Papillae. J Vis Exp; 100: e52860.

13. Pavlidis P, Gouveris H, Kekes G, Maurer J (2014):Electrogustometry thresholds, tongue tip vascularization, and density and morphology of the fungiform papillae in diabetes.B-ENT; 10: 271-278.

14. Proserpio C, Laureati M, Bertoli S, Battezzati A, Pagliarini E (2015): Determinants of Obesity in Italian Adults: The Role of Taste Sensitivity, Food Liking, and Food Neophobia. Chem Senses; 00: 1-8.

15. Salbe AD, DelParigi A, Pratley RE, DrewnowskiA,Tataranni PA (2004): Taste preferences and body weight changes in an obesity-prone Population. Am J ClinNutr; 79:372-378.

16. Scott J, Valentine JA, Hill CA ST,West CR (1985): Morphometric analysis of atrophic changes in human lingual epithelium in iron deficiency anaemia. J ClinPathol; 38:1025-1029.

17. Segovia C, Hutchinson I, Laing DG, Jinks AL (2002): A quantitative study of fungiform papillae and taste pore density in adults and children. Brain Res Dev Brain Res;138(2):135-146.

18. Webb J, Bolhuis DP, Cicerale S, Hayes JE, Keast R (2015): The Relationships Between Common Measurements of Taste Function. Chem Percept; 8:11-18. 